\title{
Clinical and CT Findings of Adenovirus Pneumonia in Immunocompetent Adults, a Two-center Retrospectively Observation
}

\section{Peiyao Zhang}

China-Japan Friendship Hospital Department of Radiology

Min Liu (D mikie0763@126.com )

China-Japan Friendship Hospital https://orcid.org/0000-0003-1298-4441

\section{Ling Zhang}

China-Japan Friendship Hospital Department of Radiology

\section{Xiaojuan Guo}

Beijing Chaoyang Hospital

\section{Binghuai Lu}

China-Japan Friendship Hospital

\section{Yimin Wang}

China-Japan Friendship Hospital

\section{Qingyuan Zhan}

China-Japan Friendship Hospital

\section{Research}

Keywords: Adenovirus, Pneumonia, CT, BALF

Posted Date: March 5th, 2020

DOl: https://doi.org/10.21203/rs.3.rs-16215/v1

License: (c) (i) This work is licensed under a Creative Commons Attribution 4.0 International License. Read Full License 


\section{Abstract}

Background: Adenovirus pneumonia account for $5 \%-10 \%$ of respiratory tract infections in children. However, it is rarely observed in immunocompetent patients. We sought to describe clinical and CT characteristics of community-acquired Adenovirus pneumonia in immunocompetent adults.

Methods: 20 patients with clinically diagnosed adenovirus pneumonia from two hospitals were retrospectively included from January 2018 to December 2019. Medical records and chest CT of all patients were reviewed and summarized.

Results: A total of 20 patients who were consisted of 18 men and 2 women with a median age of 36 years-old (range, 18-48). The clinical findings of patients with adenovirus pneumonia is high fever $\left(>39^{\circ} \mathrm{C}\right)$ with the normal white blood cell (WBC) count and decreased Lymphocyte and elevated C-reactive protein. 10 cases complicated with mycoplasma infection when admission. Staphylococcus epidermidis was found in blood culture of 2 patients. CT findings included consolidation in all patients, ground glass opacity (GGO) in 13 patients, and pleural effusion in 12 patients. 13 patients were mechanically ventilated and 2 patients died during hospitalization.

Conclusions: The most frequent finding of adenovirus pneumonia on CT was consolidation in the early stage. In adult patients with high fever, normal WBC count and decreased Lymphocyte, consolidation with/without GGO in the early stage may be a clue for suspecting adenovirus pneumonia.

\section{Background}

Adenovirus is a double-stranded DNA virus widely distributed in nature. It is mainly transmitted through the respiratory tract, causing not only upper respiratory tract infections but also pneumonia ${ }^{[1]}$. Adenovirus pneumonia is more common in children under 5 year old and immunocompromised people [2, 3] and may progresses rapidly to severe pneumonia, acute respiratory distress syndrome, and even death. Previous studies of adenovirus pneumonia in immunocompetent adults mostly focused on outbreaks and epidemics of adenoviral pneumonia in military camps, schools, and medical institutions ${ }^{[4]}$. In recent years, the incidence of immunocompetent adults has gradually increased and was reported to account for $1-7 \%$ of adult respiratory infections ${ }^{[4]}$. However, most cases are reported sporadically while the clinical and radiological reports of adult community-acquired adenovirus pneumonia was limited ${ }^{[5]}$.The purpose of the present study was to summary clinical and CT characteristics of community-acquired adenovirus pneumonia in immunocompetent adults.

\section{Methods}

\section{Study population}

The study protocol was approved by the institutional ethical review board. Informed consent was waived because this was a retrospective study. Patients with adenovirus pneumonia at China-Japan hospital and 
Beijing Chaoyang hospital from January 2018 to December 2019 were retrospectively included. All the patients were diagnosed by a positive nucleic acid detection for adenovirus in bronchoalveolar lavage fluid (BALF). Medical records of all patients were reviewed to obtain the clinical data of the patients, including age, sex, symptoms, laboratory and etiological findings, clinical course, and survival outcome et al. Patients who underwent in-patient CT were included. Patients who were younger than 18 years old were excluded.

\section{Chest CT and analysis}

Thoracic CT were performed ( Canon CT囚Aquilion one and Simens, SOMATOM Definition Dual Source CT, Germany) in two imaging centers using parameters as follow: calibration, $0.5 \mathrm{~mm} \times 80 \mathrm{~mm}$, tube voltage, $120 \mathrm{kVp}$; Automatic modulation tube current; $5.0 \mathrm{~mm}$ slice thickness with $5.0 \mathrm{~mm}$ gap and $1.0 \mathrm{~mm}$ slice thickness with $1.0 \mathrm{~mm}$ gap (the latter was used for multiple planar reconstruction). Imaging characters were evaluated by 2 experienced radiologists in consensus.

\section{Statistical analysis}

Statistical analysis was performed on SPSS (version 20.0, IBM Corp., Armonk, NY, USA).Continuous variables were expressed as median with interquartile range (IQR) and compared by Mann-Whitney $U$ test, and categorical variables as number (proportion) and compared by chi-square test or Fisher's exact test. All of those were two-sided test, $\mathrm{P}<0.05$ was considered statistically significant difference.

\section{Result}

\section{Clinical characteristics}

A total of 20 patients who were consisted of 18 men and 2 women with a median age of 36 (range, 18-48) years were included. Clinical characteristics of these patients were summarized in Table 1. The symptoms and signs on admission include fever $(n=20,39.2 \pm 0.80 \mathrm{C})$, dry cough $(n=6)$, diarrhea $(n=6)$, sore throat $(n=4)$, dyspnea $(n=6)$, pharyngeal congestion $(n=6)$, moist/dry rales $(n=13)$ and myalgia $(n=20)$. Moreover $\$ No patient presented with tonsil enlargement, conjunctivitis or lymphadenopathy. 10 cases complicated with mycoplasma infection and Staphylococcus Epidermidis was found in blood culture of 2 patients when admission. 14 patients took oral antibiotics before admission. An average smoking time of 12 patients was $4 \pm 2$ years. The median duration of symptom onset prior to the hospital admission was 6 days (IQR, 3-8days). 13 patients required mechanical ventilation among whom 11 patients were mechanically ventilated as soon as they were admitted to the hospital and 2 patients were mechanically ventilated within 2 days due to the rapid decrease in partial oxygen pressure after admission. 7 patients had no mechanical ventilation during hospitalization. The median length of hospital stay was 17 days $\varangle \mathrm{IQR}, 12-33$ days $₫$ while 2 patients respectively died in 7 days and 11 days after admission. Figure 1 demonstrated the season distribution of adenovirus pneumonia onset and the months with the highest incidence were August, and then February. 


\section{Laboratory findings}

Baseline laboratory findings of patients on admission were demonstrated in the table 2 . The median total WBC counts, median platelet count, cholinesterase were in normal levels, the median Lymphocyte percentage and $\mathrm{PO} 2$ decreased while the median neutrophil percentage, $\mathrm{C}$ reaction protein, procalcitonin (PCT), LDH and CK levels elevated.

Table 3 showed there was no statistically significant difference in the neutrophil percentage and $\mathrm{PO}_{2}$ between patients with and without mycoplasma infection. Moreover, no significant difference was observed in $\mathrm{PO}_{2}$ between patients with and without mechanical ventilation. However, patients with mechanical ventilation had a higher neutrophil percentage than patients without mechanical ventilation $(p=0.001)$

\section{CT findings of Adenovirus Pneumonia}

Initial CT findings of adenovirus pneumonia were indicated in table4 and 5 . The predominant CT findings were consolidation (100\%) and Ground-glass opacity (GGO) (65\%), which was unilateral or bilateral peribronchovascular and central distribution. Segmental consolidation of patients (70\%) diffusely distributed in the bilateral lung lobes, while in the remaining patients, lobular consolidation was predominantly localized in the unilateral upper or lower lung lobe since lower lobes were more commonly involved in patients with mechanical ventilation. No pure GGO were found all patients, in contrast, GGO combined with consolidation which was mostly unilateral or bilateral, was a single lobe distribution or random distribution was prone to be observed in patients with mechanical ventilation. Nodules/masses were seen in merely 2 patients with mechanical ventilation, and bronchiectasis was found in only 1 patient. No significant interstitial abnormality (interlobular septal thickening or Honeycombing sign or crazy-paving sign) was found. Unilateral Pleural effusion was only found in two-thirds patients with mechanical ventilation. In addition, pericardial effusion was presented in 4 patients with mechanical ventilation.

CT findings between patients with mycoplasma infection and those without mycoplasma infection were similar (Fig.2).Focal consolidation with GGO were the main findings in the patients without mechanical ventilation. In contrast, for the patients requiring mechanical ventilation, predominant bilateral or unilateral lobular consolidation with GGO were indicated in 8 patients' initial CT scans (Fig.3). Five patients showed focal consolidation in initial CT scans, then the temporal CT showed rapid progression including, more extensive unilateral or bilateral consolidation with adjacent GGO, and unilateral or bilateral pleural effusion(Fig.4).

\section{Discussion}

Human adenoviruses (HAdVs) are non-enveloped, double-stranded DNA viruses and common pathogens in children and cause a variety of diseases. Community-acquired adenoviral pneumonia is prone to occur in immunocompromised hosts and, the condition may also be a complication in immunocompetent 
patients ${ }^{[6]}$. There were no immunocompromised cases in the current study, the most common symptoms and signs were fever, myalgia and dry cough, which was consistent with previous reports ${ }^{[4,7]}$. Most patients had the high fever. The patients also indicated some other symptoms as other studies reported ${ }^{[8]}$, including dyspnea, diarrhea, pharyngeal congestion and sore throat. Tristan et al revealed that significant co-morbidity was uncommon among adenovirus pneumonia patients ${ }^{[8]}$. There were 10 patients in the present study showed combined mycoplasma infection which was similar with a previous study as it reported that co-infection of mycoplasma pneumonia was common in patients with adenovirus pneumonia ${ }^{[9]}$. Bacterial co-infections were identified in $28.5 \%(47 / 165)$ of adenovirus pneumonia patients ${ }^{[1]}$, in contrast, it was found in $10 \%$ cases in current study. However, there is no accurate association between co-infection and the adenovirus respiratory infections. Previous studies of pediatric patients showed that a peak positive rate of adenovirus infections occurred during March-April or spring ${ }^{[10,11]}$. Yao et al ${ }^{[12]}$ reported Human adenovirus among hospitalized children was detected in every month throughout the study period from April 2017 to March 2018, peaking in August 2017. In current study, summer and winter were peak season of immunocompetent adult adenovirus pneumonia, which was partially similar with another study that reported seasonal peak for adenovirus infections in winter and spring in immunocompetent patients ${ }^{[13]}$.

Consistent with previous studies, the baseline laboratory findings including a normal total white cell count, decreased lymphocyte and increased $C$ reaction protein ${ }^{[9,14]}$ which suggest virus infection. Meanwhile, the elevated procalcitonin and neutrophil percentage may relate with coexisting mycoplasma or staphylococcus epidermidis. Furthermore, we also found that there were elevated LDH and CK levels in patients with adenovirus pneumonia which was similar to a study on an adenovirus-associated outbreak in a Military Training Facility ${ }^{[4]}$. Another important finding, patients with mechanical ventilation had a higher neutrophil percentage than patients without mechanical ventilation, suggesting patients suffering from co-infection may require more respiratory support.

In the current 20-patient series, the most frequent finding on CT is consolidation in all cases, whereas most observed was focal or lobar consolidation with lobular or peribronchovascular distribution. Patchy pure GGO were not common, but consolidation with surrounding GGO is the other common CT finding. In immunocompetent military trainees ${ }^{[1,15]}$, consolidation was more commonly found than GGO in adenovirus pneumonia patients and the study of community-acquired adenovirus pneumonia revealed that consolidation, GGOs and pleural effusions were the most common findings in severe cases ${ }^{[16]}{ }^{[17]}$. A research of Adenovirus pneumonia of 5 immunocompromised patients showed the extensive groundglass opacities with or without consolidation were the main findings on $\mathrm{HRCT}^{[18]}$. In contrast, hyperinflation and lobar atelectasis were reported commonly in infants and children ${ }^{[19]}$. These suggests that consolidation with / without GGO in the early stage may be a key clue to suspecting adenovirus pneumonia for immunocompetent patients, although it resembles bacterial pneumonia. A case report and review of the literature reported bilateral interstitial infiltrates were the most common CXR in adenovirus 
pneumonia of immunocompetent adults ${ }^{[8]}$. However, diffuse interstitial abnormalities were not found in the present study, which probably because it was masked by consolidation that appeared early [20]

Gu et al ${ }^{[21]}$ suggested that adenovirus pneumonia of patients with acute respiratory distress syndrome (ARDS) usually present rapid development of bilateral, mutilobar consolidation and patchy GGO which was more severe than patients without ARDS. In the current study, focal or subsegmental consolidation was more frequent in patients without mechanical ventilation, while patients with mechanical ventilation commonly indicated initial predominant lung consolidation or progress rapidly from initial focal consolidation to lobar consolidation, suggesting that patients required mechanical ventilation may be

more prone to indicate intra-pulmonary abnormalities in CT scans. Consistent with previous studies ${ }^{[13,21]}$ , $60 \%$ of this group of cases demonstrated pleural effusion and $20 \%$ of patients indicated pericardial effusion, which may be related to more serious abnormalities.

That all Adenovirus pneumonia were confirmed with a positive finding of Adenovirus in bronchoalveolar lavage fluid is the strength of our research, however, our study has several limitations. The number of cases is small, which may partly be explained by the low incidence of adenovirus pneumonia in immunocompetent adults, however, we could miss more cases who didn't have bronchoalveolar lavage or mild cases. Because it is retrospective study, the scanning intervals of CT for patients were different leading to the dynamic changes on CT was unclear and adenovirus typing were not available leading to the relation of the clinical and CT findings with adenovirus typing remains unknown.

\section{Conclusion}

In conclusion, adenovirus pneumonia in immunocompetent patients manifested usually as significant consolidation with or without ground-glass opacities on CT. These findings are not specific for adenovirus pneumonia, but in adult patients with high fever, normal WBC count and decreased lymphocyte, consolidation with/without GGO on CT in the early stage may be a clue for suspecting adenovirus pneumonia.

\section{Abbreviations}

CT: Computed tomography

WBC: White blood cell

GGO: Ground glass opacity

BALF: Bronchoalveolar lavage fluid

IQR: Interquartile range

PCT: Procalcitonin 
LDH: Lactate dehydrogenase

CK: Creatine kinase

ARDS: Respiratory distress syndrome

\section{Declarations}

\section{Acknowledgements}

Not applicable.

\section{Funding}

Not applicable.

\section{Authors' Contributions}

Conception and Design (LM, ZPY, LBH, WYM, ZQY), analysis and Interpretation (ZPY, LM, GXJ, ZL, LBH, WYM, ZQY), drafting the manuscript (ZPY), reviewing and editing the manuscript (LM). All authors read and approved the final manuscript.

\section{Availability of data and materials}

The datasets used and/or analysed during the current study are available from the corresponding author on reasonable request.

\section{Ethics approval and consent to participate}

All procedures were approved by the institutional ethical review board of China-Japan Friendship Hospital (2017-25).

\section{Consent for publication}

Not applicable.

\section{Competing interests}

The authors declare that they have no competing interests.

\section{References}

\section{References}

[1] Yoon H, Jhun BW, Kim H, Yoo H, Park SB. Characteristics of Adenovirus Pneumonia in Korean Military Personnel, 2012-2016. J Korean Med Sci. 2017. 32(2): 287-295. 
[2] Lu L, Zhong H, Su L, et al. Detection and Molecular Characterization of Human Adenovirus Infections among Hospitalized Children with Acute Diarrhea in Shanghai, China, 2006-2011. Can J Infect Dis Med Microbiol. 2017. 2017: 9304830.

[3] Shen CF, Wang SM, Wang JR, Hu YS, Ho TS, Liu CC. Comparative study of clinical and epidemiological characteristics of major pediatric adenovirus epidemics in southern Taiwan. BMC Infect Dis. 2019. 19(1): 681.

[4] Hwang SM, Park DE, Yang YI, et al. Outbreak of febrile respiratory illness caused by adenovirus at a South Korean military training facility: clinical and radiological characteristics of adenovirus pneumonia. Jpn J Infect Dis. 2013. 66(5): 359-65.

[5] Cha MJ, Chung MJ, Lee KS, et al. Clinical Features and Radiological Findings of Adenovirus Pneumonia Associated with Progression to Acute Respiratory Distress Syndrome: A Single Center Study in 19 Adult Patients. Korean J Radiol. 2016. 17(6): 940-949.

[6] Kim EA, Lee KS, Primack SL, et al. Viral pneumonias in adults: radiologic and pathologic findings. Radiographics. 2002. 22 Spec No: S137-49.

[7] Vento TJ, Prakash V, Murray CK, et al. Pneumonia in military trainees: a comparison study based on adenovirus serotype 14 infection. J Infect Dis. 2011. 203(10): 1388-95.

[8] Clark TW, Fleet DH, Wiselka MJ. Severe community-acquired adenovirus pneumonia in an immunocompetent 44-year-old woman: a case report and review of the literature. J Med Case Rep. 2011. 5: 259.

[9] Cao B, Huang GH, Pu ZH, et al. Emergence of community-acquired adenovirus type 55 as a cause of community-onset pneumonia. Chest. 2014. 145(1): 79-86.

[10] Li Y, Zhou W, Zhao Y, et al. Molecular typing and epidemiology profiles of human adenovirus infection among paediatric patients with severe acute respiratory infection in China. PLoS One. 2015. 10(4): e0123234.

[11] Zhao MC, Guo YH, Qiu FZ, et al. Molecular and clinical characterization of human adenovirus associated with acute respiratory tract infection in hospitalized children. J Clin Virol. 2020. 123: 104254.

[12] Yao LH, Wang C, Wei TL, Wang H, Ma FL, Zheng LS. Human adenovirus among hospitalized children with respiratory tract infections in Beijing, China, 2017-2018. Virol J. 2019. 16(1): 78.

[13] Tan D, Fu Y, Xu J, et al. Severe adenovirus community-acquired pneumonia in immunocompetent adults: chest radiographic and CT findings. J Thorac Dis. 2016. 8(5): 848-54.

[14] Chen WW, Nie WM, Xu W, et al. Cross-sectional study of the relationship of peripheral blood cell profiles with severity of infection by adenovirus type 55. BMC Infect Dis. 2014. 14: 147. 
[15] Park CK, Kwon H, Park JY. Thin-section computed tomography findings in 104 immunocompetent patients with adenovirus pneumonia. Acta Radiol. 2017. 58(8): 937-943.

[16] Tan D, Zhu H, Fu Y, et al. Severe Community-Acquired Pneumonia Caused by Human Adenovirus in Immunocompetent Adults: A Multicenter Case Series. PLoS One. 2016. 11(3): e0151199.

[17] Brosch L, Tchandja J, Marconi V, et al. Adenovirus serotype 14 pneumonia at a basic military training site in the United States, spring 2007: a case series. Mil Med. 2009. 174(12): 1295-9.

[18] Chong S, Lee KS, Kim TS, Chung MJ, Chung MP, Han J. Adenovirus pneumonia in adults:

radiographic and high-resolution CT findings in five patients. AJR Am J Roentgenol. 2006. 186(5): 128893.

[19] Koo HJ, Lim S, Choe J, Choi SH, Sung H, Do KH. Radiographic and CT Features of Viral Pneumonia. Radiographics. 2018. 38(3): 719-739.

[20] Ou ZY, Zeng QY, Wang FH, et al. Retrospective study of adenovirus in autopsied pulmonary tissue of pediatric fatal pneumonia in South China. BMC Infect Dis. 2008. 8: 122.

[21] Gu L, Liu Z, Li X, et al. Severe community-acquired pneumonia caused by adenovirus type 11 in immunocompetent adults in Beijing. J Clin Virol. 2012. 54(4): 295-301.

\section{Tables}

Table 1. Clinical characteristics of patients with Adenovirus Pneumonia 


\begin{tabular}{|c|c|}
\hline Patient Characteristics & Cases \\
\hline Age, median (range), year & $36(18-48)$ \\
\hline Sex,$M / F$ & $18 / 2$ \\
\hline Fever $\left(>37.5^{\circ} \mathrm{C}\right)$ & $20(100 \%)$ \\
\hline High Fever $\left(>39^{\circ} \mathrm{C}\right)$ & $11(55 \%)$ \\
\hline Dry cough & $16(80 \%)$ \\
\hline Myalgia & $20(100 \%)$ \\
\hline Pharyngeal congestion & $6(30 \%)$ \\
\hline Dyspnea & $6(30 \%)$ \\
\hline Diarrhea & $6(30 \%)$ \\
\hline Sore throat & $4(20 \%)$ \\
\hline Dry rales & $12(60 \%)$ \\
\hline Moist & $1(5 \%)$ \\
\hline Combined mycoplasma infection & $10(50 \%)$ \\
\hline Hypertension & $2(10 \%)$ \\
\hline Gallstone & $1(5 \%)$ \\
\hline Kidney stone & $1(5 \%)$ \\
\hline Gout & $1(5 \%)$ \\
\hline Smoking & $12(60 \%)$ \\
\hline Oral antibiotic history before admission & $14(70 \%)$ \\
\hline Outcome( Improve/Death) & $18 / 2$ \\
\hline
\end{tabular}

Table 2. Initial laboratory findings for patients Adenovirus Pneumonia on admission

\begin{tabular}{|c|c|c|}
\hline Laboratory finding & Median (IQR) & Reference range \\
\hline Total WBC count $\left(\times 10^{9}\right.$ cells $\left./ \mathrm{L}\right)$ & 6.47ロ2.65-11.03凸 & $3.5-9.5$ \\
\hline Neutrophil percentage (\%) & $79.9(56.7-95.3)$ & $40-75$ \\
\hline Lymphocyte percentage (\%) & $17.0(14.0-22.0)$ & $20-50$ \\
\hline Platelet count $\left(\times 10^{9}\right.$ cells $\left./ \mathrm{L}\right)$ & $144(76-233)$ & $125-350$ \\
\hline $\mathrm{C}$ reaction protein $\square \mathrm{mg} / \mathrm{L} \square$ & $54.04(7.36-157.7 \square$ & $0-10$ \\
\hline PCT (ng/L口(n =13) & $3.52(0.27-27.63)$ & $0-0.5$ \\
\hline $\mathrm{LDH} \square \mathrm{U} / \mathrm{L} \square(\mathrm{n}=17)$ & $387(135-2843)$ & $100-250$ \\
\hline $\mathrm{CK} \square \mathrm{U} / \mathrm{L} \square(\mathrm{n}=17)$ & $313(34-4627)$ & $26-200$ \\
\hline Cholinesterase $\square \mathrm{U} / \mathrm{L} \square(\mathrm{n}=5)$ & $4347(347-6778)$ & $5400-13200$ \\
\hline $\mathrm{PO}_{2} \square \mathrm{mmHg} \square$ & $75.3(37.5-104)$ & $95-100$ \\
\hline
\end{tabular}


IQR, interquartile range; WBC, white blood cell; PCT, procalcitonin; LDH, lactate dehydrogenase; CK, creatine kinase.

Table 3. Comparison of laboratory findings of patients with adenovirus pneumonia

\begin{tabular}{ccccccc}
\hline Variables & Neutrophil percentage, $\%$ & P & PO2 घmmHg & P \\
& $\square 75$ & $40-75$ & & $\square 95$ & $95-100$ & \\
\cline { 2 - 7 } Mycoplasma infection(+) & 5 & 5 & 0.350 & 9 & 1 & 1.000 \\
Mycoplasma infection(-) & 8 & 2 & & 8 & 2 & \\
Mechanical ventilation(+) & 12 & 1 & $0.001^{*}$ & 13 & 0 & 0.350 \\
Mechanical ventilation(-) & 1 & 6 & & 6 & 1 & \\
\hline
\end{tabular}

$\mathrm{P}$ values were calculated using the chi-square test, $\mathrm{p} \otimes 0.05$,the difference was statistically significant.

${ }^{*}$ The difference was statistically significant.

Table 4. CT findings of patients with Adenovirus Pneumonia on admission

\begin{tabular}{|c|c|}
\hline CT findings & case \\
\hline Consolidation & $20(100 \%)$ \\
\hline Ground-glass opacity & $13(65 \%)$ \\
\hline Nodules/Masses & $2(10 \%)$ \\
\hline Bronchiectasis & $1(5 \%)$ \\
\hline Hyperinflation & 0 \\
\hline Tree-in-Bud & 0 \\
\hline Crazy Paving sign & 0 \\
\hline Septal thickening & 0 \\
\hline Honeycombing sign & 0 \\
\hline Halo sign & 0 \\
\hline Anti-halo sign & 0 \\
\hline Cavity & 0 \\
\hline lobar atelectasis & 0 \\
\hline Pleural effusion & $12(60 \%)$ \\
\hline Pericardial effusion & $4(20 \%)$ \\
\hline Pneumothorax & 0 \\
\hline Mediastinal emphysema & 0 \\
\hline Lymph node enlargement & 0 \\
\hline
\end{tabular}


Table 5. Parenchymal abnormalities in adenovirus pneumonia patients

\begin{tabular}{|c|c|c|c|}
\hline CT findings & $\operatorname{Total}(\mathrm{n}=20)$ & Group1 $(n=13)$ & Group2 $(\mathrm{n}=7)$ \\
\hline \multicolumn{4}{|l|}{ Parenchymal opacities } \\
\hline Consolidation & $20(100 \%)$ & $13(100 \%)$ & $7(100 \%)$ \\
\hline GGO & $13(65 \%)$ & $10(77 \%)$ & $3(43 \%)$ \\
\hline Consolidation with GGO & $13(65 \%)$ & $10(77 \%)$ & $3(43 \%)$ \\
\hline Nodular opacities & $2(10 \%)$ & $2(15 \%)$ & 0 \\
\hline \multicolumn{4}{|l|}{ Laterality } \\
\hline Bilateral & $14(70 \%)$ & $8(62 \%)$ & $6(86 \%)$ \\
\hline Unilateral & $6(30 \%)$ & $5(38 \%)$ & $1(14 \%)$ \\
\hline \multicolumn{4}{|l|}{ Zone } \\
\hline Middle & $1(5 \%)$ & $1(8 \%)$ & 0 \\
\hline All & $5(25 \%)$ & $4(31 \%)$ & $1(14 \%)$ \\
\hline Upper & $4(20 \%)$ & $1(8 \%)$ & $3(43 \%)$ \\
\hline Lower & $10(50 \%)$ & $7(54 \%)$ & $3(43 \%)$ \\
\hline \multicolumn{4}{|l|}{ Distribution } \\
\hline Central & $20(100 \%)$ & $13(100 \%)$ & $7(100 \%)$ \\
\hline Peripheral & 0 & 0 & 0 \\
\hline Peribronchovascular & $20(100 \%)$ & $13(100 \%)$ & $7(100 \%)$ \\
\hline \multicolumn{4}{|l|}{ Extent } \\
\hline Focal & $10(50 \%)$ & $4(31 \%)$ & $6(86 \%)$ \\
\hline Multifocal & $10(50 \%)$ & $9(69 \%)$ & $1(14 \%)$ \\
\hline Plueral effusion & $12(60 \%)$ & $11(85 \%)$ & $1(14 \%)$ \\
\hline Bilateral & $4(20 \%)$ & $3(23 \%)$ & $1(14 \%)$ \\
\hline Unilateral & $8(40 \%)$ & $8(62 \%)$ & 0 \\
\hline Pericardial effusion & $4(20 \%)$ & $4(100 \%)$ & 0 \\
\hline
\end{tabular}

Abbrevations: CT computed tomography; GGO, ground-glass opacity. Group1, patients with mechanical ventilation; Group2, patients without mechanical ventilation.

\section{Figures}




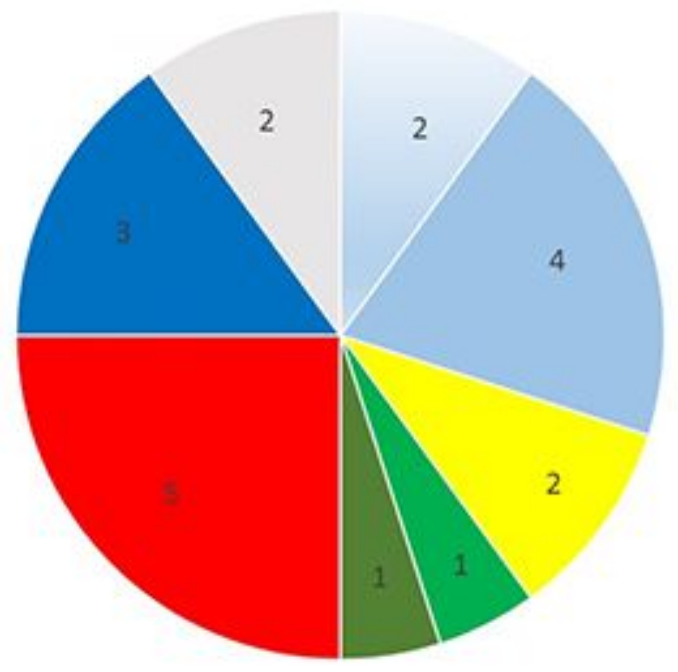

- January = February March = April = May = August = December November

\section{Figure 1}

Onset time of the 20 patients with adenovirus pneumonia.

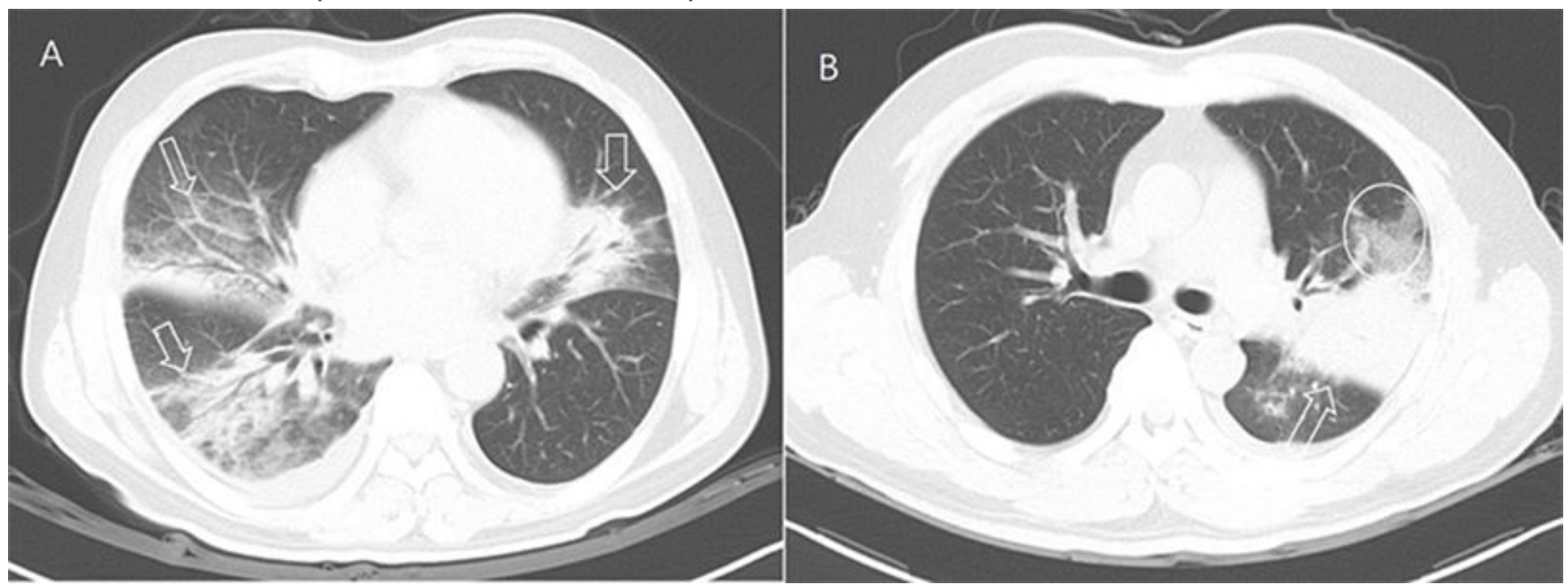

Figure 2

(A) A 48-year-old man with adenovirus pneumonia without mycoplasma infection, multifocal bilateral consolidation (arrows) was found in the picture; (B) A 46-year-old man with adenovirus pneumonia combined with mycoplasma infection, focal consolidation (arrow) with surrounding GGO (circle) was shown in the left lower lobe. 


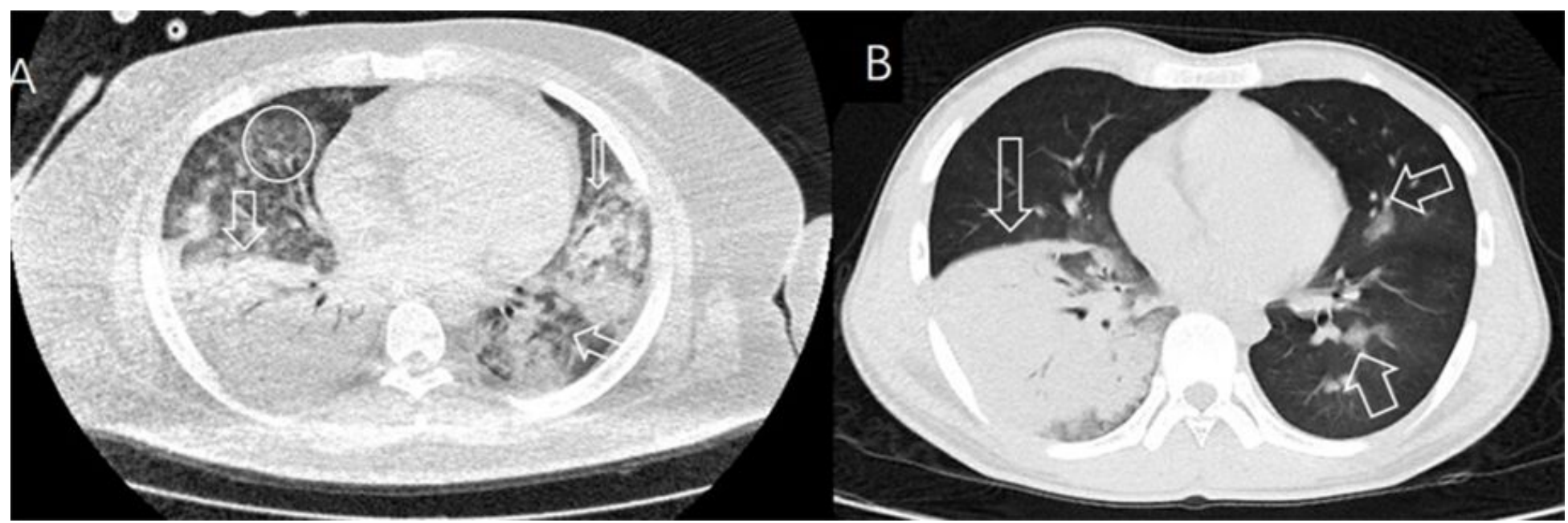

Figure 3

CT scans of the 2 cases with adenovirus pneumonia but without mycoplasma infection, and mechanical ventilation was performed in the patients. (A) Initial CT scan of a 39-year-old man showed predominant consolidation(arrows) with adjacent GGO(circle) in the bilateral lung lobes; (B) Initial CT scans of an 18year-old man indicated multifocal consolidation in the bilateral lung lobes (arrows).

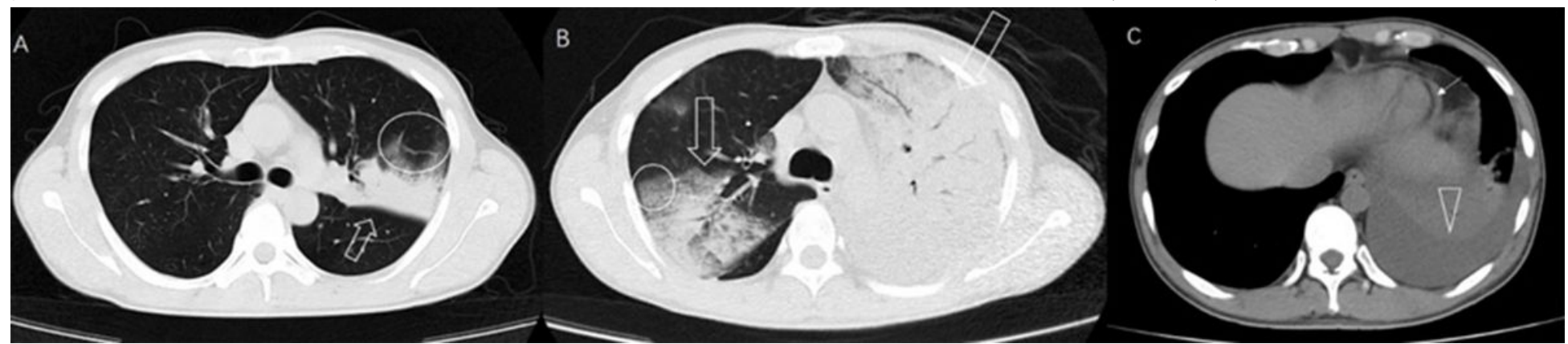

Figure 4

CT scans of a 41-year-old man with adenovirus pneumonia combined with mycoplasma infection, and mechanical ventilation was performed in the patient. (A) Initial CT scan showed focal consolidation (arrows) with adjacent GGO (circles) in the left lower lobe; (B) Rapid progression of consolidation in both lung zones (arrows) with surrounding GGO (circles) after 5 days; (C) Left pleural effusion was also observed. 